Mitteilungen der Österreichischen Geographischen Gesellschaft, 162. Jg., S. 539-544

(Annals of the Austrian Geographical Society, Vol. 162, pp. 539-544)

Wien (Vienna) 2020, https://doi.org/10.1553/moegg162s539

\title{
Martin Seger zum 80. Geburtstag
}

\author{
Doris WASTL-WALTER, Bern*
}

mit 2 Abb. im Text

Martin SEGER ist 80 Jahre alt. Es ist kaum zu glauben. Seine Vitalität und sein Arbeitseifer sind ungebrochen. Drei Tage nach seinem 80. Geburtstag fand am Institut für Geographie und Regionalforschung der Alpen-Adria-Universität in Klagenfurt am Wörthersee die Präsentation seines „Opus Magnum“ statt, des Prachtbands „Österreich-Raum und Gesellschaft. Vermessung der Landschaft, Porträts der Bundesländer " mit 648 Seiten, 450 Grafiken und Diagrammen, 30 doppelseitigen Karten und mehr als tausend wunderschönen und charakteristischen Fotos. Eine unglaubliche Leistung!

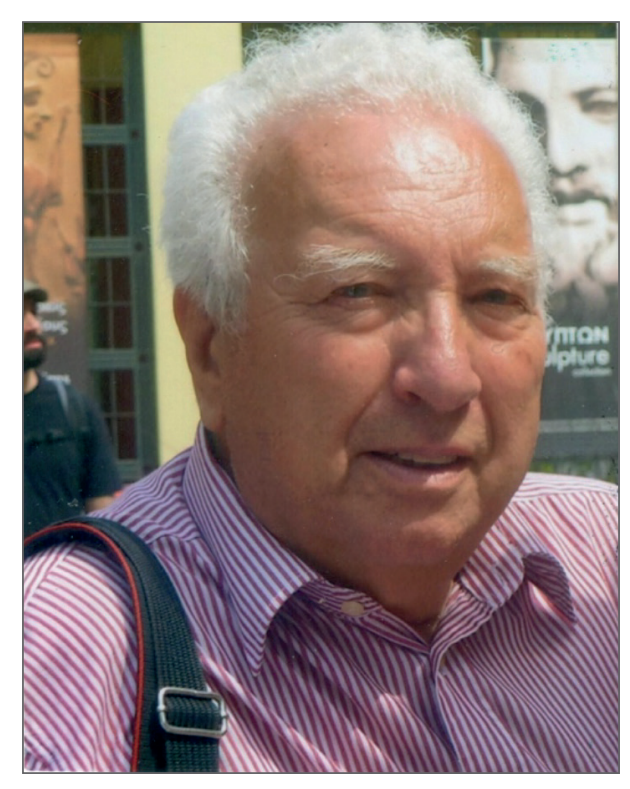

Martin Seger (Foto: Fritz Palencsar)

Martin SEGER wurde am 27. Jänner 1940 als Ältester von drei Söhnen geboren und wuchs in der Hinterbrühl in Mödling in einem ehemaligen Forsthaus der Liechtenstein'schen Forstverwaltung auf. Sein Vater war ein bekannter Lehrer, Maler und Grafiker, sodass er schon früh mit Fragen der künstlerischen visuellen Gestaltung vertraut wurde. SEGER hat an der Universität Wien bei den Professoren KüHnelt und HöPler Biologie und bei Prof. Spreitzer und Prof. BobeK Geographie

\footnotetext{
* Prof. em. Mag. Dr. Doris WAStL-Walter, Professorin Emerita für Kulturgeographie, Universität Bern, Geo-
} graphisches Institut, Hallerstraße 12, CH-3012 Bern. - E-Mail: wastl@giub.unibe.ch. 
studiert und damit wurde die Grundlage für eine Karriere als ordentlicher Universitätsprofessor für Physische Geographie und Geoökologie gelegt. Letztere, die bei seiner Berufung an die Universität Klagenfurt noch eine sehr junge Disziplin war, erforderte Kenntnisse der biologischen und ökologischen Zusammenhänge, wie Martin SEGER sie vorwies.

Während des Studiums hat er Elisabeth HeInZE kennengelernt, die 1967 seine Frau wurde und ihn all die Jahre seither im Hintergrund massgeblich unterstützt hat und sich um die Familie mit den drei Kindern Christoph, Barbara und Bernhard kümmerte. Sie haben beide bei Prof. BoвeK am damaligen Geographischen Institut in Wien dissertiert: Elisabeth HeINZE über St.Pölten 1967 und Martin Seger mit einer sozialgeographischen Dissertation über den Raum Mödling (publiziert1972), mit dem Abschluss als Dr. phil. im Jahr 1969.

Parallel dazu unterrichtete Martin SEgER nach Abschluss des Lehramtsstudiums 1965 (als Mag. rer. nat.) in Mödling als Lektor der Wiener Universitäten am „Vorstudienlehrgang“ des Österreichischen Akademischen Austauschdienstes (OeAD) für ausländische Studierende, wo er auch persische Studenten kennenlernte, die sein Interesse für den Orient weckten und ihn dann auch zu sich in den Iran einluden. Rasch faszinierte ihn die orientalische Stadt und insbesondere die zweipolige Entwicklung der Metropole Teheran, womit der Ausgangspunkt für seine Habilitationsschrift gelegt war, an der er von 1973 bis 1977 als Universitätsassistent bei Prof. Elisabeth LiCHTENBERGER am Wiener Institut für Geographie arbeitete. Darüber hinaus unterrichtete Martin SEGER in Klagenfurt seit der Einrichtung des Geographie-Studiums an der neu gegründeten Universität, das heißt, ab dem Wintersemester 1975/76. 1976 erfolgte dann in Wien die Habilitation für Geographie mit der Habilitationsschrift „Teheran. Eine stadtgeographische Studie“.

Schon jung wurden sein wissenschaftliches Potenzial und die Relevanz seiner Arbeiten erkannt. Bereits 1971 erhielt er den Wissenschaftspreis des Landes Niederösterreich als Förderungspreis sowie 1974 für seine stadtgeographischen Forschungen den Camillo-Sitte-Preis für junge Wissenschaftler der Technischen Universität Wien.

Innerhalb seines wissenschaftlichen Werks von fast 200 Publikationen gibt es mehrere Schwerpunkte, die mit geo- und landschaftsökologischer Umwelt- und Regionalforschung, sozialgeographischer Stadt- und Grenzforschung und technisch-naturwissenschaftlicher Fernerkundung und Kartographie als Methodenpionier und Künstler nur grob umrissen sind. Diese Themenfelder wurden aus einer innovativen Forschungsperspektive, aber gleichzeitig auch didaktisch und praxisorientiert aufgegriffen.

\section{Martin Seger, ein Umwelt- und Regionalforscher}

Biologie und Geographie haben die Grundlagen gelegt für das in den 1970er Jahren sehr innovative Konzept der geo- und landschaftsökologischen Umweltforschung, das Martin SEGER an der neu geschaffenen Professur für Physische Geographie und Geoökologie an der Universität Klagenfurt ab 1978 umgesetzt hat. Schon vorher hat er in seiner vegetationskundlichen Studie über den Eichkogel 1976 Biologie und Geographie zusammengeführt. Im ersten großen gemeinsamen Projekt der wissenschaftlichen Mitarbeiterinnen und Mitarbeiter des Instituts in Klagenfurt, einer umfassenden Studie zur Abgrenzung des Nationalparks Hohe Tauern in Kärnten (1982, 1984), hat er den landschaftsökologischen Teil abgedeckt (1987).

In Klagenfurt boten sich mit den neuen technischen Voraussetzungen für Fernerkundung und der Unterstützung durch den Assistenten Peter MANDL neue Rahmenbedingungen für ökologische und praxisorientierte Umweltforschung, die u. a. in den „Forschungen zur Umweltsituation in Klagenfurt“ (1985) und „Stadt und Umwelt. Arbeiten zur Grünraumsituation in Villach» (1988) publiziert wurden. 
Als Mitte der 1980er Jahre das Waldsterben zum großen Thema wurde, konnte Martin SEGER seine Kenntnisse in der Auswertung von Farbinfrarot-Luftbildern und die daraus abgeleitete landschaftsökologische Modellbildung in ein Sofortprogramm gegen das Waldsterben, in Folgeprogramme und schließlich in den Schwerpunkt „Fernerkundung“ des „Fonds zur Förderung der wissenschaftlichen Forschung“ (FWF) einbringen (1988). Diese Kenntnisse wurden auch außerhalb Österreichs nachgefragt und so kam es auch zu einer umfangreichen Landnutzungsstudie am Peloponnes (1985).

In erster Linie war es Martin SEGER aber wichtig, für Österreich und besonders für das Land Kärnten einen praxis- und planungsrelevanten geographischen Beitrag zu leisten und er erstellte mehrere Studien zum Waldzustand in Kärntner Regionen (1993, 1994). Diese und viele andere, thematisch unterschiedliche, aber immer planungsrelevante Arbeiten, waren sehr relevant für Kärnten und 2011 wurde ihm daher für seine Forschung der Kulturpreis des Landes Kärnten verliehen.

\section{Martin Seger, ein Stadt- und Grenzforscher}

Martin SEgERs stadtgeographische Interessen haben sich zuerst in der Dissertation über Mödling und dann in der Habilitation über Teheran (publiziert 1978) manifestiert. Dort hat er das bekannte Modell der orientalischen Stadt unter westlichem Einfluss entwickelt und Stadtkarten erstellt, die Heinz Petritsch gezeichnet hat und die immer noch als Grundlage der Stadtplanung anerkannt sind. Teheran hat ihn auch später noch wiederholt beschäftigt, u. a. mit der Publikation 2004 über Miyanabad, ein Stiftungsdorf im Weichbild der Metropole Teheran. Es liegt aber auch seine zentralörtliche Typisierung der iranischen Städte vor. SEgER wurde immer wieder nach Teheran zu Vorträgen und als Experte eingeladen und auch heute arbeitet er an Luftbildern von Teheran zur neuesten Stadt- und Agglomerationsentwicklung.

Ab 1999 haben sich zudem seine zahlreichen Aufenthalte in Istanbul mit Fritz PALENCSAR in einer Reihe von Karten und Artikeln sowie 2006 in dem Buch „Istanbul. Metropole zwischen den Kontinenten" niedergeschlagen. Auch über diese Millionenstadt am Schnittpunkt von Europa und Asien hat er immer wieder geforscht und publiziert, wie zum Beispiel 2010 und 2011 über die Stadtentwicklung und Segregation im Großraum Istanbul. Aber auch andere islamisch geprägte Städte und deren Strukturen haben ihn beschäftigt, wie eine Analyse der Strukturelemente der Stadt Kairo 1986 zeigt.

Nicht nur orientalische Städte haben Martin SEGER fasziniert. sondern auch Städte im Umbruch bzw. im Transformationsprozess, und die gemeinsame Feldarbeit mit der Autorin 1990 in Halle an der Saale kurz nach der Wende war Grundlage für den Aufsatz „Die sozialistische Stadt in Mitteleuropa. Der Modellfall Halle an der Saale. Zustand und Struktur am Ende einer Epoche“. Die Feldforschung in Kenya, bei der SEGER auch überfallen wurde, mündete in der Publikation über „Nairobi. Struktur und Funktion einer postkolonialen Primate City“, die 2000 wieder aufgenommen wurde.

Martin SEGER war immer bereit, neue, spannende Themen aufzugreifen und sie mit seiner umfassenden Kompetenz weiter zu entwickeln. Als Beispiel sei hier nur die in den späten 1980er Jahren begonnene Forschung am ehemaligen Eisernen Vorhang im Südburgenland und in den angrenzenden ungarischen Komitaten genannt. Gemeinsam mit Kolleginnen und Kollegen der Ungarischen Akademie der Wissenschaften und der Autorin führte er einen multimethodischen Regionalvergleich auf mehreren Maßstabsebenen sowohl mittels einer statistischen Analyse als auch mithilfe von Luftbildanalysen der Landnutzung zum Zeitpunkt der Öffnung der Grenze durch, was eine einmalige Momentaufnahme zu einem historisch herausragenden Zeitpunkt erlaubte (SEGER und BeluszKY, Hrsg., 1993: Bruchlinie Eiserner Vorhang: Regionalentwicklung im österreichisch-ungarischen Grenzraum). 


\section{Martin Seger, ein Methodenpionier und Künstler}

Neben der Stadtgeographie war es vor allem die Regional- und Umweltforschung, die Martin SEGER methodisch innovativ mit Fernerkundung oder GIS betrieben hat und wo er seine humangeographischen und geoökologischen Kompetenzen produktiv und oft anwendungsorientiert zusammengeführt hat. Mit dem Beginn seiner Tätigkeit in Klagenfurt und der neuen technischen Ausstattung des damaligen Instituts für Geographie erschienen die ersten Arbeiten zur Fernerkundung, wie 1981 ein Artikel gemeinsam mit Peter MANDL oder 1982 ein Beitrag zum Verständnis von Satelliten- und anderen Fernerkundungsbildern.

Seit 1981 veröffentlichte er laufend Publikationen, in denen dargestellt wird, wie die modernen GIS- und Fernerkundungstechniken zur Gewinnung neuer Erkenntnisse beitragen können, etwa zur Gliederung der Großlandschaften Österreichs nach ökologisch orientierten Landschaftstypen oder zur „Differenzierung des österreichischen Alpenraumes nach dominanten Nutzungen“ (2011). Martin SEgER ging mit diesen Methoden aber auch über Österreich hinaus, wie etwa bei den Beiträgen „Geographical Dimensions of the Alps-Adriatic Region“ (2006) und „Die Region Alpen-Adria in Thematischen Karten“ (2009) ersichtlich ist. Immer wieder publizierte er auch über Afrika, wie zum Beispiel über Landnutzung und Vegetationstypen in Uganda 2007.

Sowohl in der Kartographie wie in der Didaktik, aber auch bei den an ein breites Publikum gerichteten Monographien kommt sein künstlerisches Talent zum Tragen. Auch im Unterricht haben seine Skizzen und Karten immer rasch klar gemacht, was mit Worten viel mühsamer zu beschreiben gewesen wäre. Und seine wunderschönen Fotos sind eine Bereicherung für jeden Text.

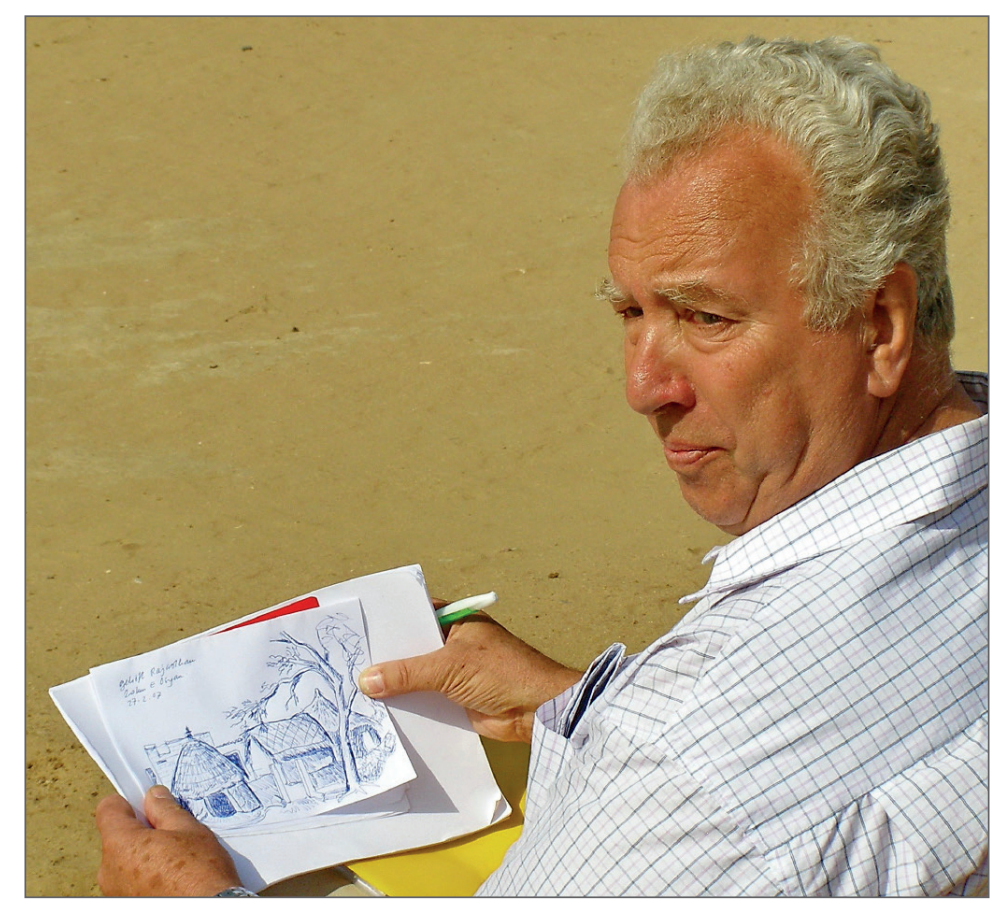

Martin Seger im Jahr 2007 beim Erstellen einer Skizze eines Gehöfts bei Nagaur in Rajasthan (Foto: Fritz PALENCSAR) 


\section{Martin Seger, ein Forscher und Lehrer}

Martin SEGERs beeindruckende Forschungsleistung wurde mehrfach ausgezeichnet: 1971 erhielt er den Wissenschaftspreis des Landes Niederösterreich, 1974 den Camillo-Sitte Preis der TU Wien, 2010 das Große Ehrenzeichen des Landes Kärnten, 2011 den Kärntner Landeskulturpreis und zuletzt 2015 die Franz-von-Hauer-Medaille der Österreichischen Geographischen Gesellschaft (ÖGG), die nach 2006 erstmalig wieder verliehen wurde. Seit 1993 ist SEGER auch korrespondierendes Mitglied der Österreichischen Akademie der Wissenschaften.

Darüber hinaus ist er aber auch ein begnadeter Lehrer und hat mehrere fachdidaktische Arbeiten publiziert. Dazu zählen das Schulbuch: „Österreich: Raum - Gesellschaft - Wirtschaft“ (gem. m. Wolfgang SitTe, 1984) und eine Reihe von Karten im Hölzel-Oberstufenatlas für Gymnasien (1981).

An der Universität Klagenfurt wurden Generationen von Studierenden von seinem Geographiekonzept geprägt; sie erzählen sich aber auch immer noch bewundernd, wie er mit der linken Hand ein Tafelbild zeichnete und es gleichzeitig mit der rechten beschriftete. Legendär sind auch die zahlreichen Exkursionen, die zwar anstrengend, aber auch ungemein lehrreich und unvergesslich waren. Hier konnte Martin Seger, oft unterstützt von Fritz PALENCSAR, mit seinem breiten Wissen und seiner Fähigkeit, Sachverhalte zu verknüpfen und zu kontextualisieren, aber auch sie plausibel und klar zu vermitteln, immer wieder brillieren.

\section{Martin Seger, ein Fachvertreter und Entscheidungsträger}

Im Jahr 1977 wurde Martin SEGER Gastdozent am Institut für Geographie der Universität Klagenfurt und 1978 ordentlicher Universitätsprofessor an der Lehrkanzel Geographie II. Gemeinsam mit Prof. Dr. Bruno BACKÉ baute er das Geographie-Institut auf und war wiederholt bis zu seiner Emeritierung Institutsvorstand. Er hat dort gemeinsam mit Bruno BACKÉ eine wegweisende und sehr moderne Geographie eingeführt, war viele Jahre Mitherausgeber der „Klagenfurter Geographischen Schriften“ und hat sich unermüdlich und uneingeschränkt für das Institut eingesetzt. Dass dieses heute unhinterfragt und respektiert in der österreichischen geographischen Community und an der Alpen-Adria-Universität Klagenfurt seinen Platz hat, ist weitgehend sein Verdienst.

Von 1993 bis 2008 übte Martin Seger auch die Funktion des Schriftleiters der „Mitteilungen der Österreichischen Geographischen Gesellschaft“ (MÖGG) aus. Er war damit der längstdienende Schriftleiter in der Nachkriegszeit und prägte das einzige geographische, für den „Social Science Citation Index“ (SSCI) referenzierte Fachjournal Österreichs nachhaltig.

Von 1998 bis 2020 war Martin SEGER für viele Jahre auch Obmann des „Österreichischen Nationalkomitees“ der Internationalen Geographischen Union (IGU) bei der Österreichischen Akademie der Wissenschaften (ÖAW) und hat die österreichische Geographie bei vielen Sitzungen und Abstimmungen entsprechend vertreten.

\section{Martin Seger, ein brillanter und vielseitiger Geograph}

All sein Wissen und seine künstlerische Begabung hat Martin SEgER auch in zwei Bücher investiert, die nicht nur von seiner Kompetenz in Kartographie und Fernerkundung, sondern auch von seinen künstlerischen Fähigkeiten, insbesondere der Fotografie, geprägt sind. Der Band „Kärnten, Landschaftsräume - Lebensräume" mit 492 Seiten, zahlreichen thematischen Karten und Fotos, erschienen 2010, war rasch vergriffen. Das neue im Herbst 2019 veröffentlichte Buch „Österreich 
- Raum und Gesellschaft. Vermessung der Landschaft, Porträts der Bundesländer " mit 648 Seiten, 30 doppelseitigen Karten zur Landesstruktur Österreichs und einer umfangreichen, mehr als tausend Bilder umfassenden Fotodokumentation regionaler Lebensräume widmet auch jeweils zirka 40 Seiten jedem Bundesland.

Die Österreichische Geographische Gesellschaft hat diese Publikation mitherausgegeben und namhafte Mitglieder haben Martin SEgER bei seiner Arbeit unterstützt. Mit diesem umfassenden, vielfältigen, empirisch fundierten und konzeptionell und methodisch innovativen Werk hat Martin SEGER eine moderne und beispielgebende Regionalgeographie publiziert, die weit über den Kreis der Geographinnen und Geographen hinaus Anklang und Interesse findet. Damit hat er dem Fach wiederum einen großen Dienst erwiesen.

Mit seinem unermüdlichen Einsatz für die Geographie als Institution in Österreich und den vielen Geographinnen und Geographen, die er ausgebildet hat, seiner Aufbauleistung am Institut für Geographie und Regionalforschung der Universität Klagenfurt über 30 Jahre und seinem andauernden Einsatz für die Österreichische Geographische Gesellschaft als Schriftleiter ihrer internationalen wissenschaftlichen Fachzeitschrift, der „Mitteilungen“ (MÖGG) über 15 Jahre hat Martin SEGER das Fach Geographie vorbildlich und nachhaltig geprägt.

Lieber Martin! Die österreichische Geographische Gesellschaft und zahlreiche Kolleginnen und Kollegen im In- und Ausland gratulieren Dir zu Deinem großen Geburtstag und wünschen dir weiterhin viel Freude und Gesundheit. Ad multos annos mirabilis!

\section{Literaturhinweise (frühere Beiträge über Martin SEGER)}

BÄTZING W. (2010): Martin Seger - 70 Jahre. In: Mitteilungen der Österreichischen Geographischen Gesellschaft, 152, S. 355-359.

Borsdorf A. (2000): Martin Seger 60 Jahre. Der Schriftleiter der MÖGG vollendete das 6. Lebensjahrzehnt. In: Mitteilungen der Österreichischen Geographischen Gesellschaft, 142, S. $355-360$.

Borsdorf A. (2000): Laudatio. In: Palencsar F. (Hrsg.): Festschrift Martin Seger zum 60. Geburtstag. Klagenfurt: Institut für Geographie und Regionalforschung, S. vii-xiv (= Klagenfurter Geographische Schriften, 18).

Wastl-Walter D. (2016): Verleihung der Franz-von-Hauer-Medaille an Martin Seger. In: Mitteilungen der Österreichischen Geographischen Gesellschaft, 158, S. 378-381. 